\title{
Simple renal cysts
}

\author{
J. E. HALE \\ F.R.C.S. \\ M. NAUnTON MORGAN \\ M.Chir., F.R.C.S.
}

Surgical Unit, Westminster Hospital, London, S.W.1

\section{Introduction}

Simple renal cyst is a benign condition usually discovered as an incidental pyelographic finding. These cysts, however, are of more than academic interest for, on occasions, complications within the cyst occur to produce unusual symptoms, which make diagnosis difficult. In addition, the co-existence of a cyst and a carcinoma in the same kidney in approximately $2 \%$ of all renal cysts surgically removed has been reported (Clarke, 1956a).

A certain diagnosis can only be made by surgical exploration, although with the use of sophisticated radiological methods, which include tomography and selective renal angiography, the accuracy of differentiating a cyst from carcinoma now approaches 95\% (Hotchkiss \& Sammans, 1965).

In this paper the incidence, aetiology, complications and treatment of solitary renal cysts are discussed and relevant publications reviewed. A series of thirty-two consecutive renal cysts seen at Westminster Hospital is presented.

\section{Incidence}

The incidence of the simple renal cyst varies with criteria accepted, and small cysts of less than $2 \mathrm{~cm}$ in diameter, often multiple, are a common postmortem finding in patients over 50 years of age (Heptinstall, 1966). The simple cyst of surgical interest is, however, usually more than $5 \mathrm{~cm}$ in diameter and, unlike the small cysts, is rare, Fish (1939) only finding thirty-two cases out of 12,000 urological admissions. Simple cysts are more often found in the lower pole and are usually solitary (Braasch \& Hendrick, 1944). Confusion with polycystic disease may occur when numerous simple cysts are present, but even when multiple, their number remains finite. Simple cysts are rare in infants and children, a fact that suggests an acquired rather than a congenital origin (Allen, 1962). Rare types of benign renal cysts include dermoid cysts (Valentine, 1929), lymphatic cysts (Henthorne,
1938) and hydatid cysts (Raffii \& Dutz, 1967). The increased use of diagnostic radiology will lead to the discovery of more simple renal cysts, and raise the problem of whether a symptom-free radiological finding should be treated.

\section{Pathology}

The simple renal cyst is lined by a single layer of cuboid cells resembling tubular epithelium. It contains a thin straw-coloured fluid with the chemical features of a transudate (Clarke, Hurwitz \& Dubinsky, 1956b). When the fluid is blood-stained the co-existence of a carcinoma has been reported to be as high as $30 \%$ (Lowsley, 1955). Many such cysts, however, are probably degenerating carcinomas, but even when a true cyst contains clear fluid, coincidental carcinoma may occasionally be found (Khorsand, 1965). It has been proposed by Schiller (1944) that there may be a direct relationship between cysts of the kidney and renal carcinoma. He suggested that extensive epithelial proliferation of cystic epithelium may progress to malignant change.

The pathogenesis of simple renal cyst is not known. Experimental obstruction of the ureter or a renal papilla may result in transient dilatation of tubules and tubular atrophy. Hepler (1930) experimentally produced epithelial-lined cysts by combining cauterization of a renal papilla with ligation of a segmental artery. The formation of a simple cyst, therefore, appears to depend on at least two factors, intra-renal obstruction together with ischaemia.

\section{Clinical features}

The majority of renal cysts are asymptomatic, being discovered incidentally on intravenous pyelogram. Most cysts are placed superficially and expand away from the kidney and can, on occasions, reach sufficient size to press on neighbouring structures, such as the stomach, to produce dyspepsia (Shivers \& Axilrod, 1953). Extremely large renal cysts, containing as much as 12 litres of fluid, 
have been recorded (Carling, 1934). When complications occur in renal cysts, the symptoms are often unusual and never pathognomonic, so that diagnosis may be difficult. Complications which have been reported, include rupture (Clarke et al., 1956a) either spontaneous or from trauma, infection (Limjoco \& Strauch, 1966), and rarely haematuria (Allen, 1962). Kreutzmann (1947) recorded two cases of hypertension associated with a solitary renal cyst, in one of which excision was followed by a satisfactorily maintained fall in blood pressure. Rarely, the clinical picture will be that of renal carcinoma which has arisen in a simple cyst (Rehm, Taylor \& Taylor, 1961).

\section{Present series}

The main clinical features of the solitary renal cyst can best be demonstrated by considering thirty-two consecutive cases seen between 1953 and 1967 at Westminster Hospital. There were sixteen males and sixteen females in the series and the majority were discovered on a routine intravenous pyelogram performed for investigation of persistent urinary symptoms. The age of the patients ranged from 20 to 79 years, but the majority occurred in the fifth decade (Fig. 1). Other investigations included non-selective or selective aortography followed by direct puncture and aspiration of the cyst under direct radiological control in ten patients. Cysts recurred in two patients who were treated either by a repeated aspiration (Case 4) or surgical

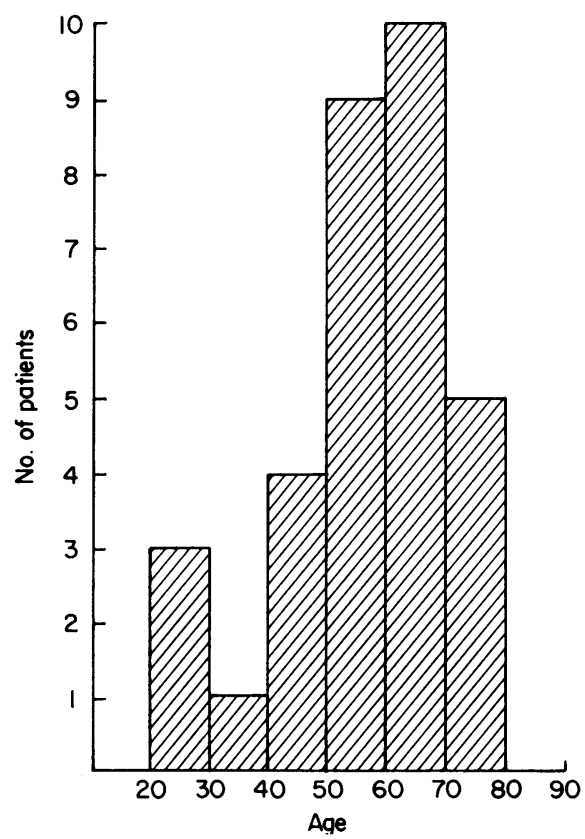

FIg. 1. Age distribution of thirty-two renal cysts.
TABle 1. Presenting findings in thirty-two simple renal cysts

\begin{tabular}{lc}
\hline Clinical feature & $\begin{array}{c}\text { No. of } \\
\text { patients }\end{array}$ \\
\hline Loin pain & 11 \\
Hypertension & 7 \\
Urinary infection & 6 \\
Haematuria & 5 \\
Abdominal mass & 3 \\
Total & 32 \\
\hline
\end{tabular}

exploration (Case 1). The major presenting symptoms (Table 1) were persistent loin pain, haematuria and recurrent urinary tract infection. Investigation of hypertension led to the discovery of seven cysts and one of these underwent decapsulation with no effective reduction post-operatively in the blood pressure. Three patients presented with an abdominal mass, which was discovered either by the family doctor in one case, or by the patient. The length of history was usually in months, but occasionally, as in Case 1, in years, In four patients complications occurred in the cysts, and these are reported in detail.

\section{Case reports}

Case 1

Female patient, aged 53, was first seen in 1964 with a 6-year history of dyspepsia and intermittern vomiting. Barium meal examination was normal: She was treated with antacids but the following year symptoms recurred, and abdominal examination revealed a palpable mass in the right loin. Intravenous pyelogram showed a mass in the right upper pole and a selective arteriogram was reported as showing an avascular mass, thought to be a cyst. Direct puncture under radiological control was performed and $320 \mathrm{ml}$ of straw-coloured fluid aspirated. In August 1967 the original symptoms recurred and she complained of vomiting after meals. On examination a mass in the right loin was palpated and intravenous pyelography (Fig. 2) confirmed that the cyst had re-formed. At operation a large cyst in the upper pole of the right kidney containing clear fluid was de-capped. One year later the patient was symptom-free.

\section{Case 2}

Female, aged 51, with several years' history of cystitis was referred for investigation of haematuria. A mass in the lower pole of the right kidney was noted on the intravenous pyelogram. A right selective arteriogram (Fig. 3) showed an avascular area which was thought due to a renal cyst in the lower pole of the right kidney, and an unsuccessful attempt was made to aspirate the suspected cyst. 


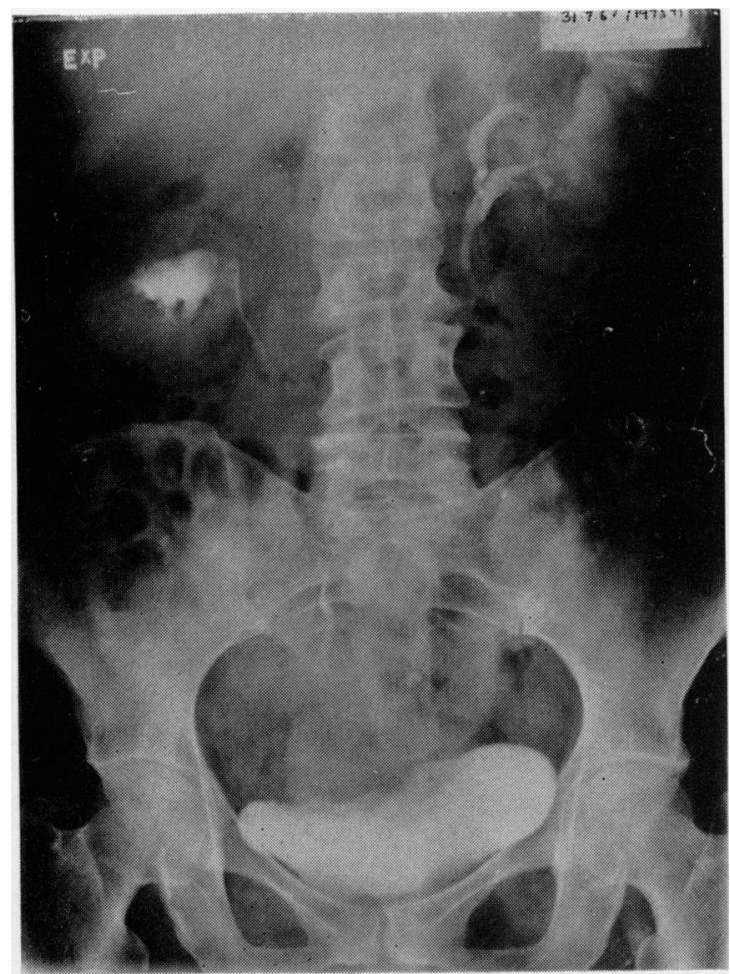

FIG. 2. Renal cyst right kidney upper pole.

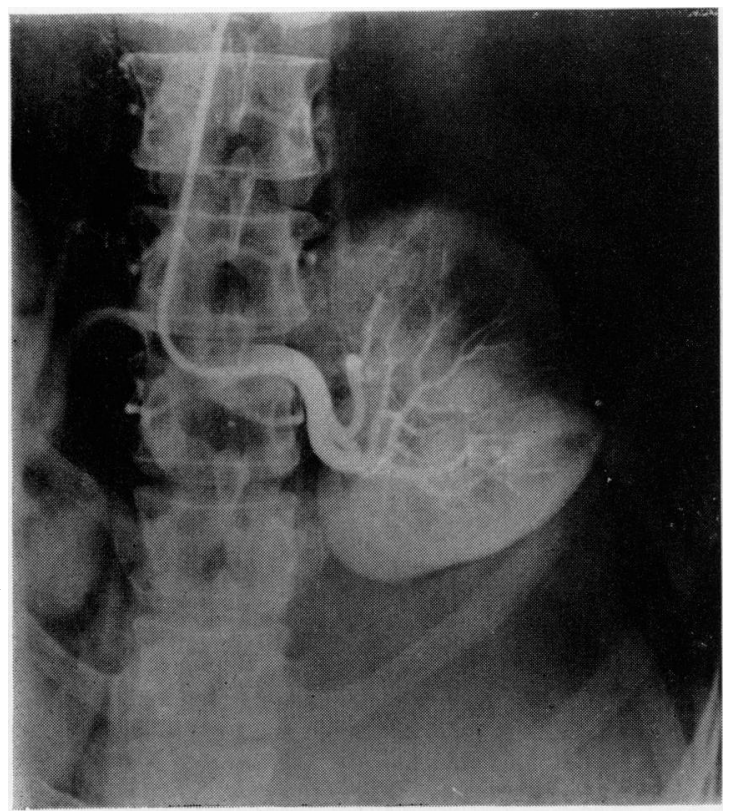

FIG. 3. Selective right renal arteriogram showing avascular area in the lower pole.
One month later the patient was admitted to another hospital with severe loin pain, and a diagnosis of infected renal cyst was again made, and a course of antibiotics prescribed. Her symptoms failed to resolve and on re-admission to the Westminster Hospital the kidney was explored. At operation a large cyst, $10 \mathrm{~cm}$ in diameter, was found in the lower pole of the right kidney. The cyst was decapped with diathermy and found to contain oldblood-clot. Frozen section of the cyst wall showed no evidence of malignancy, but a paraffin section subsequently revealed a renal carcinoma. One week later a right nephrectomy was performed. The patient received a course of external irradiation, but 8 months following operation she complained of severe back pain. A gamma scan of the spine revealed spinal deposits, which were irradiated.

\section{Case 3}

A female aged 81 years was seen with a severe haematemaesis, the haemoglobin being $7 \cdot 7 \mathrm{~g} / 100$ $\mathrm{ml}$ on admission. There was no recent history of dyspepsia. Clinical examination of the abdomen revealed a hard palpable mass which was thought to be the gall bladder. Jaundice was not present and the cholecystogram showed a functioning gall bladder full of non-opaque calculi.

A barium meal was reported to show no intrinsic abnormality of stomach or duodenum, but the latter was noted to be very distorted by an extrinsic mass (Fig. 4). At operation, the duodenum was found to be stretched over an enormous cyst arising from the upper pole of an otherwise normal kidney. The stomach and duodenum were normal. The renal cyst was excised with diathermy and a cholecystectomy was performed. The patient 3 months later was well and symptom free.

\section{Case 4}

A female of 62 years in 1964 with a 2-year history of discomfort in the left loin and occasional vomiting. There were no urinary symptoms. On examination the left kidney was palpable. The intravenous pyelogram revealed a mass in the upper pole of the left kidney. A selective renal arteriogram (Fig. 5a) confirmed the presence of a cyst which was punctured and clear straw-coloured fluid obtained. A specimen was sent for cytological examination and contrast medium was then injected to outline the smooth cyst wall (Fig. 5b). The patient remained symptomfree until 1969 when she developed pain in the left loin. Investigations as before confirmed that the left renal cyst had recurred and this was again punctured and $35 \mathrm{ml}$ of clear fluid aspirated.

\section{Diagnosis}

Radiological methods have so improved that 


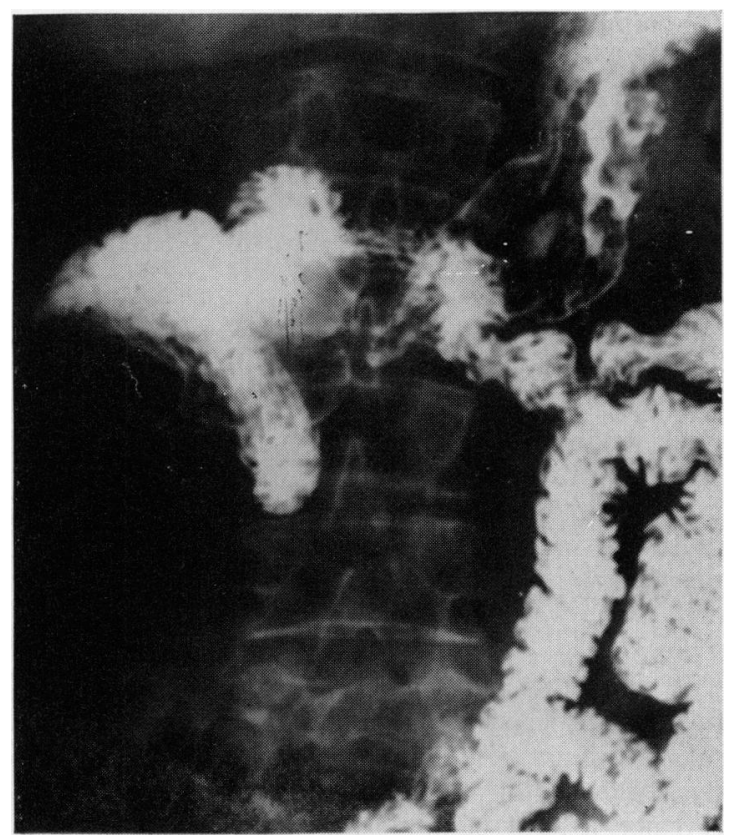

FIG. 4. Barium meal showing the duodenum distorted by a renal cyst in the right upper pole.

most space-occupying lesions in the kidney can be diagnosed accurately without surgery, although, even with the most advanced investigations, diagnosis cannot be certain. Abdominal X-rays may show a spherical soft-tissue swelling of the kidney and sometimes calcification in the cyst is seen
(Strauss \& Welt, 1963) although this is more suggestive of a neoplasm. Intravenous pyelography alone is too inaccurate (Prather, 1957), but with renal angiography, especially if selective angiography is used in combination with tomography, a renal $\stackrel{0}{\rightarrow}$ cyst can be diagnosed with a high degree of accuracy, $\Rightarrow$ (Frimann-Dahl, 1963). The use of an intra-arterial injection of minute amounts of adrenalin to produce $\frac{\bar{\omega}}{\frac{}{5}}$ temporary vasoconstriction of normal renal arteries, $\stackrel{\mathbb{D}}{\square}$ leaving tumour vessels relatively unaffected, is also said to increase diagnostic precision (Abrams, $\vec{\circ}$ Boijsen \& Borgstrom, 1962).

When there is little reasonable doubt of diagnosis, $\overrightarrow{\vec{\omega}}$ the cyst can be punctured under radiological $\stackrel{\Omega}{\circ}$ control. Percutaneous puncture and aspiration of $\stackrel{\circ}{\circ}$ renal cysts, first described by Dean in 1939, was 3 . modified by Lindblom (1946), who aspirated the cyst $\dot{\text { or }}$ contents and then injected contrast material to $i r$ outline the cyst wall. Vestby (1967) has further $\mathcal{O}^{-}$ improved this method by injecting both air and $/$ \% water-soluble contrast material into the cyst, after aspiration.

Two main objections, however, have been raised $\vec{D}$ to the puncture of renal cysts. First, in spite of the $\mathbb{\mathbb { D }}$ high degree of accuracy, using specialized radio- $\overparen{\varnothing}$ logical technique, diagnosis without exploration 3 can never be certain. Although the risk of puncturs $\frac{\mathbb{D}}{-}$ ing a malignant tumour is small, seeding of tumot $\overrightarrow{0}$ cells along the aspiration track remains a potentia danger, and puncture of a hydatid cyst could fatal. The risk of aspiration must be weighed against that following surgical exploration which, in the elderly, is considerable. Plaine \& Hinman (1965)
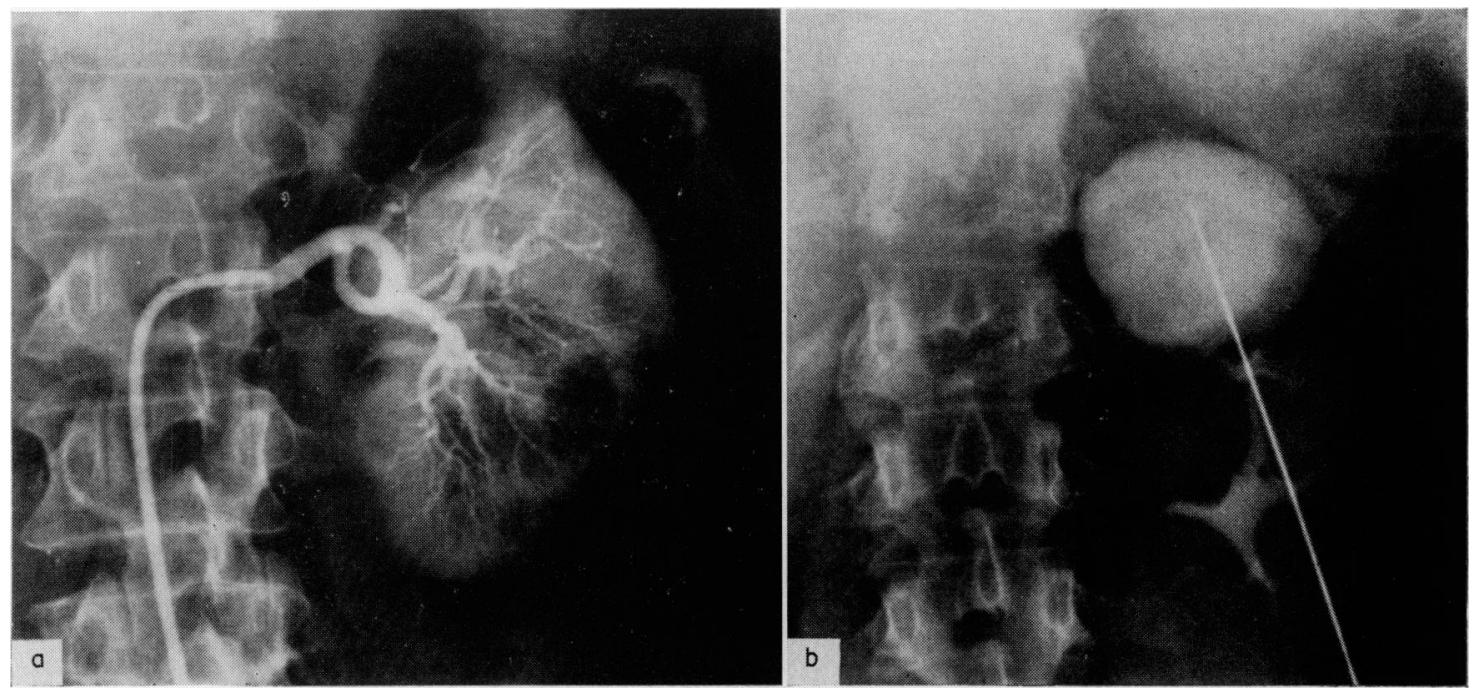

FIG. 5. (a) Selective left renal arteriogram showing an avascular area in the upper pole, thought to be a cyst. (b) Confirmed by puncture and aspiration. 
reported serious complications, including mortality in $2.4 \%$ of 102 renal cysts explored. Secondly, a malignant tumour may undergo cystic degeneration and can be overlooked if treatment is confined to aspiration alone. The internal contours of the cyst must be carefully examined to exclude malignant change within the cyst.

Clinically, renal carcinoma is far more likely to present with haematuria, intermittent pyrexia and a raised erythrocyte sedimentation rate. In addition, a raised lactic dehydrogenase in the urine has been claimed by some workers (Wacker \& Dorfman, 1962) to be useful in the differentiation of malignant from benign space-occupying lesions. Others (Riggins \& Kiser, 1963) were unable to confirm this. Schapiro, Wellington \& Gomick (1968) have reported that urinary $\beta$-glucuronidase activity is increased in patients with renal carcinoma.

The simple solitary renal cyst is a relatively rare finding, and because of possible complications and the problem in diagnosis, it would seem reasonable to treat such renal cysts, even in the absence of symptoms. Provided facilities exist for accurate radiological study, and these should include selective renal arteriography, most cysts can be accurately diagnosed. Where there is no clinical evidence of malignancy and a renal arteriogram indicates a renal cyst, this can be punctured under direct radiological control, the contents aspirated and a sample of fluid sent for analysis and cytology. Radio-opaque contrast material is injected into the cyst and the cystogram obtained carefully examined. Failure to aspirate the cyst, or the presence of blood-stained aspirate, should lead to immediate exploration of the kidney. There should be no delay as in the second case reported.

In recent years opinion has been divided regarding the therapeutic effect of renal cyst puncture. According to some authors even large cysts may completely disappear after percutaneous aspiration (De Weerd, 1962). However, Wahlguist \& Grumstedt (1966) suggested that radiographic disappearance, or reduction of size in the cyst following puncture, is only temporary. Vestby (1967) reported the treatment of twenty patients using a non-absorbable medium Pantopaque. He suggested that the oily contrast medium produced a certain degree of foreign body reaction and that this was a significant factor in achieving a good therapeutic result.

Puncture and aspiration of a simple renal cyst for confirmation of diagnosis is a safe and accurate procedure, but exploration of the solitary renal cyst should always be considered in the fit patient, especially when radiological facilities are inadequate. Clarke et al. (1956a) considered that operation was always best in the fit patient and that only poor risk patients should be subjected to aspiration. At operation every effort is made to preserve renal tissue and simple decapsulation of the cyst is usually adequate (Glaser, 1952). The excised cyst wall must be examined by frozen section to exclude malignancy, and the residual cyst wall diathermied. This procedure is relatively simple, and nephrectomy is rarely necessary.

\section{Acknowledgments}

We wish to thank the Consultant Staff and the Department of Medical Photography and Radiology of Westminster Hospital for their helpful co-operation.

\section{References}

Abrams, H.L., Boijsen, E. \& Borgstrom, K.E. (1962) Effect of epinephrine on the renal circulation. Angiographic observations. Radiology, 79, 911.

Allen, A. (1962) The kidney. Medical and Surgical Diseases. Grune \& Stratton, New York.

BraAsCH, W.F. \& Hendrick, J.A. (1944) Renal cysts, simple and otherwise. J. Urol. 51, 1.

Carling, E.R. (1934) Large solitary cyst of renal origin. Brit. J. Surg. 22, 184.

Clarke, B.G., GoAde, W.J., JR, Rudy, H.L. \& Rockwood, L. (1956a) Differential diagnosis between cancer and solitary serous cysts of the kidney. J. Urol. 75, 922.

Clarke, B.G., Hurwitz, I.S. \& Dubinsky, E. (1956b) Solitary serous cysts of the kidney: biochemical, cytological, and histological studies. J. Urol. 75, 772.

DEAN, A.L. (1939) Treatment of solitary cyst of the kidney by aspiration. Trans. Amer. Ass. Genito-Urin. Surg. 32, 91.

DE WeERD, J.R. (1962): Percutaneous aspiration of selected expanding renal lesions. J. Urol. 87, 303.

FisH, G.W. (1939) Large solitary serous cysts of the kidney. J. Amer. med. Ass. 112, 514.

FrimanN-DaHL, J. (1963). Selective renal angiotomography. Genova, Estratto da Atti del $5^{\circ}$ Corso Internazionale Sulla Tomografia.

Glaser, S. (1952). Simple renal cysts. Brit. J. Surg. 40, 74.

Henthorne, J.C. (1938) Peripelvic lymphatic cysts of the kidney. Amer. J. Clin. Path. 8, 28.

Heptinstall, R.H. (1966) Pathology of the Kidney. Churchill, London.

HEPLER, A.B. (1930) Solitary cysts of kidney: report of seven cases and observations on pathogenesis of these cysts. Surg. Gynec. Obstet. 50, 668.

Hotchisiss, R.S. \& Sammans, B.P. (1965) Selective renal angiography. J. Urol. 93, 309.

KHORSAND, D. (1965) Carcinoma within solitary renal cysts. J. Urol. 93, 440.

KREUTZMANN, H.A.R. (1947) Hypertension associated with solitary renal cysts: report of two cases. J. Urol. 57, 467.

Limjoco, U.R. \& Strauch, A.E. (1966) Infected solitary cyst of the kidney. Report of a case and review of the literature. J. Urol. 96, 625.

LindBLOM, K. (1946) Percutaneous puncture of renal cysts and tumours. Acta Radiol. 27, 366.

LoWSLEY, O.S. (1955) Malignant cyst of the kidney. J. Urol. 74, 586.

Plaine, L.I. \& Hinman, F., JR (1965) Malignancy in asymptomatic renal masses. J. Urol. 94, 342.

Prather, G.C. (1957) Surgical treatment of serous cysts of the kidney. J. Urol. 77, 14.

RafFI, P. \& Dutz, W. (1967) Hydatid cysts of the kidney. J. Urol. 97, 815.

Rehm, R.A., TAylor, W.N. \& TAylor, J.H. (1961) Renal cysts associated with carcinoma. J. Urol. 86, 307. 
Riggins, R.S. \& Kiser, W.S. (1963) A study of lactic dehydrogenase in urine and serum of patients with urinary tract disease. J. Urol. 90, 594.

Schapiro, A., Wellington, P. \& Gomick, H. (1968) Urinary beta-glucuronidase in urological diseases of the kidneys. J. Urol. 100, 146.

Schiller, W. (1944) The so-called Grawitz tumour and its interpretation by help of a new concept of heterotopic tumours. Urol. Cutam. Rev. 48, 511.

Shivers, C.H. DeT. \& Axilrod, H.D. (1953) Solitary renal cysts. J. Urol. 69, 193.
Strauss, M.B. \& Welt, L.G. (1963) Diseases of the Kidney. Little, Brown, Boston

VAlentine, J.J. (1929) Dermoid cyst of kidney. Amer. J. Surg. 6, 93.

Vestby, G.W. (1967) Needle puncture of renal cysts. Invest. Radiol. 2, No. 6.

WACKER, W.E.C. \& DORFMAN, L.E. (1962) Urinary lactic dehydrogenase activity. Screening method for detection of cancer of kidneys and bladder. J. Amer. med. Ass. 181, 972.

WaHLQUisT, L. \& GRUMSTEDT, B. (1966) Therapeutic effect of percutaneous puncture of simple renal cyst. Acta chir. scand. 132, 340. 